\title{
Lipoprotein (a): a possible link between lipoprotein metabolism and thrombosis
}

Lipoprotein (a) was first described in 1963 by Kare Berg. It was initially considered to be a qualitative genetic marker but has subsequently been shown to be a quantitative trait. The concentration of lipoprotein (a) can vary enormously between different individuals. Epidemiological studies typically show a highly skewed distribution within a population, with most individuals having low concentrations of lipoprotein (a). Much of the interest in lipoprotein (a) stems from several clinical studies that showed a highly significant association between raised concentrations of lipoprotein (a) and susceptibility to coronary artery disease. Angiographic studies suggest that the concentration of lipoprotein (a) is a risk factor for disease in both native coronary vessels and saphenous vein bypass grafts. ${ }^{1}$ In addition, lipoprotein (a) immunoreactive material has been identified in the endothelium and intima of atherosclerotic coronary arteries.

Though lipoprotein (a) was characterised in some detail in the 1970s, interest in its structure-function relations has intensified recently. Essentially, lipoprotein (a) is a low density lipoprotein (LDL) particle that has been modified by the binding of a unique high molecular mass glycoprotein called apolipoprotein (a) to apolipoprotein $B$, the

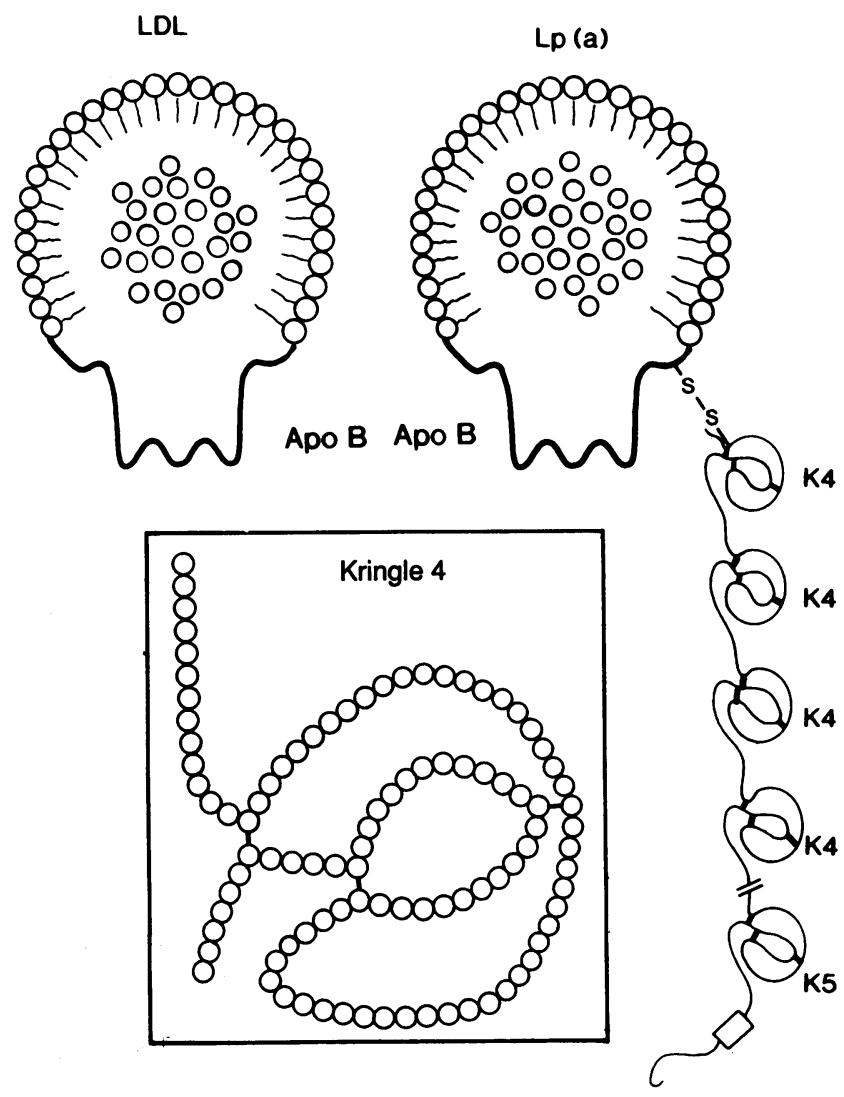

Figure 1 Structure of low density lipoprotein ( $L D L)$ and lipoprotein (a) $(L p(a))$. Lipoprotein ( $a$ ) is essentially an LDL particle modified by the binding of apolipoprotein $(a)(a p o(a))$ via a disulphide bridge to apolipoprotein $B$. [Inset] Pretzel-like structure of the kringle 4 domain in apolipoprotein $(a)$. sole protein constituent of LDL. This macromolecular complex is known as lipoprotein (a) (fig 1). Lipoprotein (a) concentrations vary considerably between individuals and it is the only major lipid risk factor with a concentration that is only slightly influenced by metabolic, endocrine, or anthropometric variables-so it remains remarkably constant within an individual. The most popular hypothesis is that a single autosomal genetic locus is the major determinant of lipoprotein (a) concentration. ${ }^{2}$ The evidence points to this locus being the gene encoding for apolipoprotein (a) itself. Apolipoprotein (a) has been shown to exist as several different isoforms-distinguished by size and mobility on gel electrophoresis. Such phenotypic variation is thought to reflect allelic polymorphisms at the apolipoprotein (a) gene locus. These allelic variants have, in turn, provided new insights into the genetics of the quantitative lipoprotein (a) trait. At least six allelic variants have been identified and an operational "null" allele postulated. There is a strong inverse relation between the mass of the differing apolipoprotein (a) isoforms and the plasma lipoprotein (a) concentrations. Thus alleles encoding for the separate apolipoprotein (a) phenotypes strongly influence the lipoprotein (a) concentration, and in individuals with the operational "null" allele lipoprotein (a) concentrations are low.

Interest in this topic was greatly stimulated by McLean et $a .^{3}$ They reported the cloning and sequencing of the apolipoprotein (a) gene. The complete primary aminoacid sequence of apolipoprotein (a) was derived from the sequenced human apolipoprotein (a) cDNA and it showed a striking homology to the structure of plasminogen-the zymogen of the fibrinolytic enzyme plasmin. Plasminogen is a single chain protein of 791 aminoacids containing several distinct structural regions. These include an $\mathrm{N}$ terminal sequence, followed by five tandemly repeated domains called kringles, each showing about $50 \%$ homology to each other, followed by a trypsin-like protease domain. The kringles are pretzel-shaped structures, stabilised by three internal disulphide bridges and are so called because they resemble a Danish cake called a kringle (fig 1 (inset)). The primary sequence of apolipoprotein (a) also contains an $\mathrm{N}$-terminal sequence one copy of plasminogen's fifth kringle and a variable copy number of the fourth kringle domain followed by a serine protease domain all of which show a high degree of homology to the equivalent regions in the plasminogen gene. In the apolipoprotein (a) cDNA sequenced by McLean $e t$ al there were 37 copies of the fourth kringle domain (fig 2). However, despite apolipoprotein (a) retaining a highly conserved serine protease domain, no latent enzymatic activity can be generated because of a crucial serine for arginine substitution at the homologous activation site.

The presence of regions of duplicated sequence retaining $100 \%$ identity within the gene suggests frequent expansion and contraction at the locus. Thus, it is possible that homologous recombination events that add or eliminate certain domains (for example kringle IV domains) at this locus are probably still occurring. This mechanism may well explain both the allelic variation at the apolipoprotein 


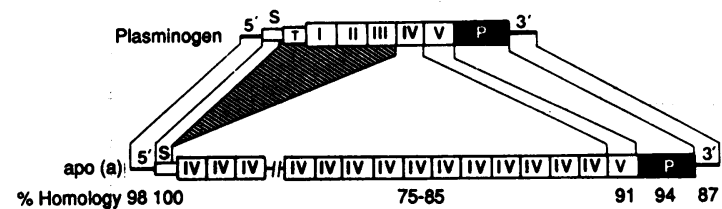

Figure 2 Comparison of the gene structures of plasminogen and apolipoprotein (a) (apo (a)). The top structure is plasminogen cDNA and the lower structure apolipoprotein (a) cDNA. Connecting lines indicate regions of homology with percentage identity between the two sequences shown at the bottom. (This illustration is derived from reference 3.) 5 ', 5 ' untranslated sequence; $S$, signal peptide; $T$, "tail" region; I, II IIII, IV, V, kringle domains; P, protease domain; $3^{\prime}, 3^{\prime}$ untranslated sequence.

(a) locus and the size variation of apolipoprotein (a) among individuals. Expression of the larger apolipoprotein (a) alleles may test the limits of the translation and transcription machinery of the cell, by mechanisms such as messenger RNA instability, decreased transcription efficiency, etc. This accords with the inverse correlation between lipoprotein (a) concentrations and the size of the apolipoprotein (a) isoforms.

In general, the kringle domains serve as regulatory domains and are important for the activation of specific proteases such as plasminogen and tissue plasminogen activator. Thus, there has been considerable speculation that lipoprotein (a) may interfere with the fibrinolytic system because of its structural homology to plasminogen. In vitro data supporting this hypothesis are beginning to accumulate. Lipoprotein (a) has been shown to inhibit binding of plasminogen to its endothelial cell binding sites with a consequent reduction in generation of plasmin by tissue plasminogen activator. Lipoprotein (a) has also been shown to compete with both plasminogen and tissue plasminogen activator for fibrin binding and it attenuates both the fibrin dependent enhancement of tissue-type plasminogen activator activity and clot lysis in vitro. ${ }^{4}$ Confirmation of these effects in vivo would demonstrate that lipoprotein (a) is a vital link between lipoprotein metabolism and the thrombolytic system.

Little is known about the synthesis and catabolism of lipoprotein (a). Circumstantial evidence suggests it is synthesised by the liver because the apolipoprotein (a) cDNA was constructed from a library of hepatic RNAs. This accords with the low concentration of lipoprotein (a) in individuals with chronic hepatic disease such as cirrhosis. While lipoprotein (a) concentrations are thought to be largely determined by the apolipoprotein (a) gene locus, other gene loci may also be influential in determining concentrations. One such locus is the LDL receptor locus. There are discrepant and conflicting data about the in vitro binding and uptake of lipoprotein (a) by the LDL receptor. However, it has been shown in vivo that irrespective of apolipoprotein (a) phenotype, patients heterozygous for familial hypercholesterolaemia, a disease caused by defective LDL receptor function, have lipoprotein (a) concentrations 2.5 to three times higher than age and sex matched controls. ${ }^{5}$ In patients with familial hypercholesterolaemia there is a 10 fold increase in the risk of coronary heart disease. In such a relatively homogeneous, monogenic disease, however, the expression of this susceptibility varies between individuals and particularly between families with the condition. Some of the variability may be explained by the finding of two recent reports from London and Sweden. ${ }^{67}$ In both, over 100 patients with familial hypercholesterolaemia were subdivided on the basis of the presence or absence of clinically documented coronary heart disease. While the LDL cholesterol concen- trations were equivalent in both subgroups, patients with coronary heart disease had significantly higher lipoprotein (a) concentrations and this value was the best discriminant for the presence of coronary heart disease on both univariate and multivariate analysis. These findings have important implications for both the management and prognosis of familial hypercholesterolaemia. Indeed, they may reveal an oversimplification in the treatments designed for the hyperlipidaemias in general. The 3-hydroxy3-methylglutaryl coenzyme A reductase inhibitors, the fibric acid derivatives, and the ion-exchange resins do not significantly alter lipoprotein (a) concentrations. To date, only nicotinic acid, neomycin, and extracorporeal apheresis have been effective in reducing lipoprotein (a) concentrations. Each of these treatments has drawbacks. Furthermore, there are no published data to show any benefit in lowering lipoprotein (a) concentrations in individual patients.

Should measurement of lipoprotein (a) concentration become part of the full lipid profile and would concomitant reduction of lipoprotein (a) and LDL cholesterol concentrations have a synergistic effect on the reduction of the risk of coronary artery disease in familial hypercholesterolaemia and the hyperlipidaemias in general? Risk reduction is being studied by a two centre trial based at the Royal Postgraduate Medical School, Hammersmith, and the University Hospital of Wales, Cardiff. This trial is designed to assess whether LDL apheresis (which lowers both lipoprotein (a) and LDL cholesterol) is more beneficial in patients with familial hypercholesterolaemia than drug treatment (which does not reduce lipoprotein (a) concentrations). It is too early to recommend the routine measurement of lipoprotein (a) concentrations. Lipoprotein (a) assays need to be standardised and fully characterised to allow appropriate interpretation. An international standardisation programme is evolving. While measurement of lipoprotein (a) is likely to be more expensive than a basic lipid profile, the rationale for measuring risk factors is to estimate the risk of developing coronary artery disease. The current controversy about the precise concentrations of cholesterol at which hypolipidaemic drug treatment should be started, and the possible role of high concentrations of lipoprotein (a) in altering the thrombotic/fibrinolytic equilibrium suggest to me that lipoprotein (a) measurements will be widely available in clinical practice within the next decade.

An understanding of the role of lipoprotein (a) in atherogenesis, evaluation of the apparent synergy between lipoprotein (a) and LDL cholesterol in promoting coronary artery disease, and the assessment of the therapeutic benefits of reducing lipoprotein (a) in specific patient groups should yield results soon.

University of Wales,

ALAN REES

Heath Park,

Cardiff CF4 4XW

1 Morrisett JD, Guyton JR, Gaubatz JW, Gotto AM Jr. Lipoprotein (a): structure, metabolism and epidemiology. In: Gotto AM Jr, ed. Plasma lipoproteins. Amsterdam: Elsevier Science, 1987:129-52.

2 Utermann G. The mysteries of lipoprotein (a). Science 1989;246:904-10.

3 McLean JW, Tomlinson JE, Kuang W-J, et al. cDNA sequence of human apolipoprotein (a) is homologous to plasminogen. Nature 1987;300:132-7. apolipoprotein (a) is homologous to plasminogen. Nature 1987;300:132-7.
Loscalzo J, Weinfeld M, Fless GM, Scanu AM. Lipoprotein (a), fibrin Loscalzo J, Weinfeld M, Fless GM, Scanu AM. Lipoprotein (a), fib
binding and plasminogen activation. Arteriosclerosis 1990;10:240-5.

5 Utermann G, Hoppichler F, Dieplinger M, Seed M, Thomson G, Bowerwinkle $E$. Defects in the low density lipoprotein receptor gene affect lipoprotein (a) levels: multiplicative interaction of two gene loci associated lipoprotein (a) levels: multiplicative interaction of two gene loci associated

with premature atherosclerosis. Proc Natl Acad Sci USA 1989;86:4171-4.
Seed M, Hoppichler F, Reaveley D, et al. Relation of serum lipoprotein (a) concentration and apolipoprotein (a) phenotype to coronary artery disease in patients with familial hypercholesterolaemia. N Engl J Med 1990;332: 1494-9.

7 Wiklund O, Angelin B, Olofsson S-O, et al. Apolipoprotein (a) and ischaemic heart disease in familial hypercholesterolaemia. Lancet 1990;335:1360-3. 